Paula Valéria de Oliveira Terra

\title{
A Avaliação Social no Benefício de Prestação Continuada: o olhar dos assistentes sociais.
}

Dissertação apresentada como requisito parcial para obtenção do grau de Mestre pelo Programa de PósGraduação em Serviço Social do Departamento de Serviço Social da PUC-Rio.

Orientador: Prof ${ }^{a}$. Andréia Clapp Salvador 
Paula Valéria de Oliveira Terra

\section{A Avaliação Social no Benefício de Prestação Continuada: o olhar dos assistentes sociais.}

Dissertação apresentada como requisito parcial para obtenção do Grau de Mestre em Serviço Social pelo Programa de PósGraduação em Serviço Social da PUC-Rio. Aprovada pela Comissão Examinadora abaixo assinada.

Prof. Andréia Clapp Salvador Orientador Departamento de Serviço Social - PUC-Rio

Prof.a Inêz Terezinha Stampa Departamento de Serviço Social - PUC-Rio

Prof.a Mônica Mônica de Castro M. Senna ESS - UFF

Profá. Mônica Herz Coordenadora Setorial do Centro de Ciências Sociais - PUC-Rio 
Todos os direitos reservados. É proibida a reprodução total ou parcial do trabalho sem a autorização da universidade, da autora e do orientador.

Paula Valéria de Oliveira Terra

Graduou-se em Serviço Social pela Escola de Serviço Social da Universidade Federal Fluminense em 1997, cursou especialização em Terapia de Família pela Universidade Cândido Mendes em 2005. Atualmente integra a Coordenação da equipe de Serviço Social do Hospital Universitário Antônio Pedro/UFF.

Ficha Catalográfica

Terra, Paula Valéria de Oliveira

A avaliação social no benefício de prestação continuada: o olhar dos assistentes sociais / Paula Valéria de Oliveira Terra ; orientador: Andréia Clapp Salvador. - 2012.

113 f. : il. (color.) ; $30 \mathrm{~cm}$

Dissertação (mestrado)-Pontifícia Universidade Católica do Rio de Janeiro, Departamento de Sociologia e Política, 2012.

Inclui bibliografia

1. Sociologia - Teses. 2. Assistência social. 3. Benefício de prestação continuada. 4. Avaliação social. 5. Serviço social. I. Salvador, Andréia Clapp. II. Pontifícia Universidade Católica do Rio de Janeiro. Departamento de Sociologia e Política. III. Título. 


\section{Agradecimentos}

Ao meu amigo e Mestre Jesus, conhecido como Carpinteiro de Nazaré, que esculpiu em meu coração um sonho e me deu condições de realizá-lo.

À PUC-Rio, pelos auxílios concedidos, sem os quais este trabalho não poderia ter sido realizado.

A minha família pelo apoio, em especial a minha mãe que cuidou dos meus filhos para que eu pudesse me dedicar ao mestrado.

Aos meus filhos Lucas e Leonardo personagens motivadores da minha busca por melhores condições de vida e aprimoramento intelectual.

Às amigas Simone Paura e Edith L. M. Lago incentivadoras do meu pontapé inicial de inscrição na seleção do Mestrado e tantas outras que me incentivaram ao longo desse desafio.

A minha orientadora Prof ${ }^{a}$. Andréia Clapp que me recebeu gentilmente no segundo tempo da partida com um sorriso nos lábios, palavras de apoio e excelente direção acadêmica.

A querida Prof ${ }^{a}$. Ana Quiroga, minha orientadora até a defesa do projeto, suas contribuições e exemplo de vida foram inspiradores para a realização deste trabalho.

Aos assistentes sociais da Gex Nit/INSS que concederam as entrevistas, em especial ao apoio da Assistente Social Carminda Ramos cujo trabalho de socialização de informações tem sido essencial para a garantia de direitos. 


\section{Resumo}

Terra, Paula Valéria de Oliveira; Salvador, Andréa Clapp. A Avaliação Social no Benefício de Prestação Continuada: o olhar dos assistentes sociais. Rio de Janeiro, 2012. 113p. Dissertação de Mestrado Departamento de Serviço Social, Pontifícia Universidade Católica do Rio de Janeiro.

Este trabalho se propõe a realizar um estudo sobre a Avaliação Social para acesso ao Benefício de Prestação Continuada (BPC) e identificar a sua repercussão no fazer profissional do Assistente Social. Desde 2009 o BPC sofreu alterações significativas no processo de concessão para pessoas com deficiência. Além de mudanças na avaliação do médico perito, foi introduzida a Avaliação Social a ser realizada pelo assistente social do INSS (Instituto Nacional de Seguro Social). $\mathrm{O}$ assistente social entrou em cena como figura que traria a concepção de outro profissional além do médico, com uma visão mais ampla das questões sociais que envolvem a vida dos requerentes. Conhecer o olhar desses profissionais e investigar as possibilidades e impasses encontrados por eles na realização da avaliação social foi nosso objetivo através da realização deste estudo. Realizamos entrevistas semi-estruturadas com 7 das 14 assistentes sociais da Gerência Executiva do INSS de Niterói. Os resultados da pesquisa demonstraram que os profissionais de serviço social acreditam que a Avaliação Social propiciou uma democratização do acesso ao BPC, pois passou a contemplar vários aspectos antes deixados de lado. Contudo, as mudanças ocorridas na legislação no ano de 2011 levaram novamente para as mãos do médico o poder decisório na concessão do benefício. Além disso, foram apontadas questões relativas condições de trabalho, exigências institucionais e padronização do instrumento que rebatem no fazer profissional do Serviço Social trazendo dificuldades na realização da Avaliação Social. Estratégias e possibilidades também foram sinalizadas pelos entrevistados principalmente no que diz respeito à aproximação e articulação com a rede de serviços na garantia dos direitos dos usuários.

\section{Palavras-chave}

Assistência Social; Benefício de Prestação Continuada; Avaliação Social; Serviço Social. 


\section{Abstract}

Terra, Paula Valéria de Oliveira; Salvador, Andréa Clapp (Advisor). The Social Evaluation in Continuous Cash Benefit: the eyes of social workers. Rio de Janeiro, 2012. 113p. MSc. Dissertation - Departamento de Serviço Social, Pontifícia Universidade Católica do Rio de Janeiro.

This work intends to conduct a study on the Social Evaluation for access to the Continuous Cash Benefit (BPC), impact on the role of social worker. Since 2009 , the BPC has significantly changed the process of granting for people with disabilities. In addition to changes in the evaluation of medical expert, was introduced to Social Assessment to be conducted by a social worker at the INSS (National Social Security Institute). The social worker entered the scene as a figure who would bring the conception of another professional other than the doctor, with a broader social issues that surround the life of the applicant. Understanding the views of professionals and investigate the possibilities and dilemmas they face in carrying out social assessment is our goal with this study. We conducted semi-structured interviews with 7 of 14 social workers of the Executive Management of the INSS Niterói. The results showed that social service professionals believe that the Social Evaluation provided a democratization of access to BPC because will consider various aspects before neglected. However, changes in legislation in 2011 led again to the doctor's hands the power to decide on granting the benefit. In addition, issues were identified as working conditions, institutional requirements and standardization of the instrument that that bring to difficulties in conducting Social Evaluation. Strategies and opportunities were also flagged by the interviewees, especially with regard to the approach and coordination with the network of services to guarantee the rights of users.

\section{Keywords}

Social Assistance; Continuous Cash Benefit; Social Evaluation; Social Work 


\section{Sumário}

1. Introdução

2. A Assistência Social no Brasil e o BPC 13

2.1. Uma breve reflexão sobre a história da assistência 13

2.2. Assistência Social e as mudanças pós Constituição de 1988:

a interface com o BPC enquanto benefício da Assistência Social 18

2.3. O Benefício de Prestação Continuada 24

2.3.1. A Importância do BPC 28

2.3.2. O BPC para pessoas com deficiência 35

3. Serviço Social e Avaliação Social no BPC 46

3.1. O Serviço Social enquanto profissão e sua atuação no âmbito Previdenciário

3.2. O acesso ao Benefício de Prestação Continuada: o papel da Avaliação Social 50

3.2.1. O instrumento de Avaliação Social 51

3.3. O olhar dos Assistentes Sociais sobre a Avaliação Social $\quad 60$

3.3.1. Percepções gerais sobre a Avaliação Social 61

3.3.2. Percepções sobre o fazer profissional: o dia-a-dia do assistente social na realização da Avaliação Social $\quad 68$

3.3.3. Dois anos de Avaliação Social 79

4. Considerações Finais $\quad 86$

5 - Referências Bibliográficas 90

$\begin{array}{ll}6-\text { Anexos } & 99\end{array}$ 


\section{Listas de abreviaturas e siglas}

BPC - Benefício de Prestação Continuada

CIF - Classificação Internacional de Funcionalidade, Incapacidade e Saúde

CNAS - Conselho Nacional de Assistência Social

CRAS - Centro de referência e Centros de Referência da Assistência Social

CREAS - Centros de Referência Especializados da Assistência Social

CRESS - Conselho Regional de Serviço Social

CFESS - Conselho Federal de Serviço Social

GEX NIT - Gerência Executiva de Niterói/ INSS

HUAP - Hospital Universitário Antônio Pedro

IAP - Instituto de Aposentadoria e Pensão

INSS - Instituto Nacional de Seguro Social

LBA - Legião Brasileira de Assistência

LOAS - Lei Orgânica da Assistência Social

LOPS - Lei Orgânica da Previdência Social

MDS - Ministério de Desenvolvimento Social e Combate à Fome.

MPS - Ministério da Previdência Social

NOB - Norma Operacional Básica NOB/Suas

RMV - Renda Mensal Vitalícia

SIS - Solicitação de Informações Sociais

SUAS - Sistema Único de Assistência Social

SUS - Sistema Único de Saúde 